\title{
Verteidiger des Verfassungsstaates, Verteidigung des Staates: Gewisser Mangel an Geschlossenheit
}

Kielmansegg, Peter Graf: Die Grammatik der Freiheit. Acht Versuche über den demokratischen Verfassungsstaat, Nomos Verlagsgesellschaft, Baden-Baden 2013, 278 Seiten, € 39,-.

Bach, Maurizio (Hrsg.): Der entmachtete Leviathan. Löst sich der souveräne Staat auf? (Sonderband 5 der Zeitschrift für Politik), Nomos Verlagsgesellschaft, Baden-Baden 2013, 342 Seiten, $€ 44,-$.

Die beiden Neuerscheinungen befassen sich auf höchst unterschiedliche Weise mit dem Staat: Während Peter Graf Kielmansegg sich Gedanken um die Gegenwart und Zukunft des demokratischen Verfassungsstaates macht, geht es in dem von Maurizio Bach herausgegebenen Reader vornehmlich um die Frage, ob der Staat unter den Bedingungen der Globalisierung an Einfluss verliert oder sich gar auflöst. Gleichwohl gibt es eine Schnittmenge - die Sorge um den Erhalt des (Verfassungs-)Staates.

Das Anliegen des kleinen Kompendiums eines großen Gelehrten besteht darin, dem Leser das komplexe Gefüge des demokratischen Verfassungsstaates, die spannungsreiche Synthese aus dem Demokratie- und dem Verfassungsprinzip, anschaulich vor Augen zu führen. Der 75-jährige Mannheimer Emeritus Kielmansegg, studierter Jurist, promovierter Historiker und habilitierter Politologe, hatte vor 25 Jahren einer Auswahl seiner Schriften einen ähnlichen Titel gegeben. ${ }^{1}$ Der jetzige spielt auf eine Formel Thomas Paines an, einen der Gründerväter der Vereinigten Staaten. „Ohne Kenntnis der Grammatik der Freiheit“, so Kielmansegg unter Berufung auf Paine, „gibt es kein Leben in Freiheit“ (S. 7).

Sein „Nachdenken über die Demokratie“, dies der Titel einer anderen Aufsatzsammlung², ist von dem Willen geprägt, ihre Wurzeln und freiheitlichen Strukturprinzipien zu verdeutlichen. Die „acht Versuche“ sind wahre Lektionen: zum Begriffspaar Demokratie und Wahrheit (mit einem Votum für Wertgewissheit), zur „Quadratur des Zirkels“ (dem Spannungsverhältnis von Volkssouveränität und Amtsprinzip), zur Unentbehrlichkeit der Parteien, zur Kritik an der direkten Demokratie, zur Akzeptanz der Verfassungsgerichtsbarkeit, der „Instanz des letzten Wortes" (Josef Isensee), zum Zusammenhang von Demokratie und Marktwirtschaft, zur Einordnung des Schlüsseljahres 1989 sowie zur Zukunft des Verfassungsstaates. Damit sind keineswegs alle einschlägigen Fragen erörtert (so kommt Kielmanseggs ceterum censeo, das Demokratiedefizit der Europäischen Union ${ }^{3}$, kaum zur Sprache, auch nicht die Diskussion um Colin Crouchs „Postdemokratie“ ${ }^{4}$ oder Kenneth Minogues „demokratische Sklavenmentalität"5).

1 Vgl. Peter Graf Kielmansegg, Das Experiment der Freiheit. Zur gegenwärtigen Lage des demokratischen Verfassungsstaates, Stuttgart 1988.

2 Vgl. ders., Nachdenken über die Demokratie. Aufsätze aus einem unruhigen Jahrzehnt, Stuttgart 1980.

3 Vgl. ders., Soll von Demokratie noch die Rede sein?, in: FAZ vom 8. Juli 2011, S. 35; ders., Zwangsintegration, in: FAS vom 16. Dezember 2012, S. 44.

4 Vgl. Colin Crouch, Postdemokratie, Frankfurt am Main 2008; ders., Über das befremdliche Überleben des Neoliberalismus: Postdemokratie II, Frankfurt am Main 2011.

5 Vgl. Kenneth Minogue, Die demokratische Sklavenmentalität. Wie der Überstaat die Alltagsmoral zerstört, Waltrop / Leipzig 2013. 
Die scharfsinnigen Überlegungen regen an, wie einige Hinweise zu den drei letzten Essays zeigen mögen. Hieß es noch in den 1980er Jahren, Demokratie könne auch mit einer Planwirtschaft funktionieren, so ist davon heute fast nichts mehr zu lesen. Die Diagnose Kielmanseggs, die Demokratie sei auf Marktwirtschaft angewiesen, diese nicht unbedingt auf Demokratie, hat viel für sich, wenngleich er bezweifelt, ob Diktaturen die Einflüsse der Marktwirtschaft auf Dauer beherrschen können. Für ihn bedeutet diese ein Stück an Gewaltenteilung, höhere Leistungsfähigkeit, die Demokratien benötigen, sowie Entlastung der Politik durch den Markt, etwa bei der Einkommensverteilung. „Der demokratische Verfassungsstaat und die Marktwirtschaft stehen als Komponenten einer freiheitlichen Gesellschaftsordnung in einem Verhältnis der Komplementarität zueinander, das nicht angemessen als Über- oder Unterordnung beschrieben werden kann. [...] Wenn man eine Metapher sucht: Es ist eine Ehe, eine alles andere als einfache Ehe, aber wenn sie denn gelingt, eine beiden Partnern sehr bekömmliche Ehe. Auch für eine Ehe ist die Frage nach dem Primat nicht hilfreich" (S. 198).

Das Epochendatum 1989 signalisiert den Sieg über ein anderes Datum dieser Tragweite. Kielmansegg interpretiert die Oktoberrevolution 1917 „als ein gewaltiges Demokratieexperiment“" (S. 215). Gewiss, er stellt auf das Selbstverständnis der Revolutionäre ab, aber wäre der Terminus "gewalttätiges Diktaturexperiment" nicht treffender? Schließlich hat Wladimir I. Lenin ebenso wie Rosa Luxemburg die „Diktatur des Proletariats“ propagiert. Der eine plädierte für die Rolle einer Avantgardepartei, die andere für Massenspontaneismus. Der Begriff des „emanzipatorischen Experiments“ (S. 215) provoziert Missverständnisse, auch wenn es heißt, die Revolution sei bruchlos in eine „totalitäre Gewaltherrschaft“ (S. 220) überführt worden.

Die Perspektiven zur Zukunft des demokratischen Verfassungsstaates fallen zu Recht vorsichtig aus - der Druck durch Immigration, Demographie und ökonomischen Wettbewerb bietet Raum für verschiedene Szenarien. Kielmansegg unterscheidet zwischen der horizontalen Dimension (der Ausbreitung der Demokratie in den Staaten der Welt), der vertikalen (des „Regierens jenseits des Nationalstaates“) und der Binnendimension (dem inneren Wandel der „alten“ Demokratien angesichts zahlreicher Herausforderungen). In einem Punkt wagt sich der Autor aus der Deckung: „Je weiter wir uns vom Nationalstaat entfernen, desto mehr an demokratischer Substanz geht verloren“" (S. 254). Kosmopolitischen Visionen dürfte dies nicht gefallen.

Kielmansegg nimmt gegnerische Ansichten ernst, macht sie gar stark, baut mithin keinen Popanz auf, wendet seine Argumente hin und her, um sie nachvollziehbar zu machen. Die Zahl der (Schlüssel-)Fragen, keine suggestiven, keine rhetorischen, ist Legion. Oft ist von „teils, teils“ die Rede, von „mehr oder weniger“, von „sowohl als auch“. Die Relativierung wird relativiert, die Differenzierung differenziert - etwa bei der Frage, wie das Verhältnis von Demokratie und Wahrheit ist. Umso überzeugender ist dann Kielmanseggs von Urteilskraft getragene Position, die zudem durch ihre begriffliche Klarheit gewinnt. Dass ein Befund so ist, wie er ist, bedeutet für ihn nicht, dass er gut ist. Zuweilen bringt bereits der erste Satz den jeweiligen Sachverhalt auf den Punkt: „Der demokratische Verfassungsstaat, inzwischen etwa 200 Jahre alt, ist die erfolgsreichste Institutionalisierung politischer Freiheit in der Geschichte der Menschheit, die wir kennen“ (S. 39). Dieser ist als repräsentative Demokratie eben nicht die zweitbeste Lösung, wie Kritiker der „gemischten Verfassung“ meinen. Der Beitrag zur „Quadratur des Zirkels“ (aus dem Jahr 1985) mit seiner Rehabilitierung der verfassungsstaatlichen Komponente gehört in ein politikwissenschaftliches Lehrbuch. 
Kielmansegg, vom nüchternen Skeptizismus Tocquevilles geprägt, nicht vom naiven Optimismus Rousseaus, was etwa das Menschenbild betrifft, steht in der Tradition der amerikanischen Verfassungsväter.

Wer die jetzige Sammlung mit der vor 25 Jahren vergleicht, erkennt eine Reihe auffallender Gemeinsamkeiten. Der Fluchtpunkt des Autors: das Regelwerk der Freiheit zu erhellen. Seinerzeit, als der „real existierende Sozialismus“ eine große Gefahr für die Freiheit bildete, machte sich Kielmansegg nicht die pessimistische Diagnose Jean François Revels zu eigen, wonach die Demokratie nur ein Zwischenspiel sei. ${ }^{6}$ Heute, nach dem Zusammenbruch des „real existierenden Sozialismus“, ist er keineswegs „siegestrunken“ und, von Francis Fukuyamas optimistischer Prognose weit entfernt. ${ }^{7}$ Die Anfälligkeit für Schwächen sei erkennbar. Kielmansegg, kein Mann des wetterwendischen Zeitgeistes, mischt sich in die Politik ein, ohne das Feld der Wissenschaft zu verlassen. Was die Komposition des Bandes betrifft, so ist sie zwischen dem „Nachdenken über die Demokratie“ (unveränderter Nachdruck von Aufsätzen) und dem „Experiment der Freiheit“ (weithin Neufassung von Aufsätzen) angesiedelt. Durch die Überarbeitung der einzelnen Essays, die eigenem Bekunden nach für sich stehen (können), wollte Kielmansegg sie stärker in Beziehung zueinander setzen. Allerdings wäre auch durch den unveränderten Nachdruck die Intention des Autors deutlich geworden, zumal er nicht kurzatmiger Aktualität huldigt. Das Ganze ist in diesem Fall nicht mehr als die Summe seiner Teile. Mit einer neuen vergleichenden Einordnung zum Schluss hätte der Autor seinem Ansinnen wohl besser gedient.

Auch Maurizio Bachs Sammelband ist von wissenschaftlichem Impetus getragen. Der Herausgeber weist einleitend auf den Strukturwandel von Staatlichkeit hin. Er wirft mehr Fragen auf, als das Werk beantwortet: „Ist der Staat unter den Rahmenbedingungen unserer Gegenwartsgesellschaft an das Ende seiner Geschichte angekommen? Wurde der über Jahrhunderte quasi omnipotente Leviathan in der neuen historischen Konstellation entmachtet? Löst sich der Staat auf? Kann es einzelstaatliche Souveränität in der heutigen globalvernetzten Weltgesellschaft noch geben? Wie veränderten sich Stellung und Funktion des Staates? Ist der Nationalstaat europäischer Provenienz noch die adäquate und unumstrittene politische Regimeform der Gesellschaft? Wie steht es um die Zukunft der Demokratie und des Rechtsstaates?" (S. 8). So radikal sich manche Fragen auch ausnehmen, so beruhigt Bach den Leser jedoch schnell: Ein Ende des souveränen Staates sei nicht in Sicht.

Das ist auch die Tendenz der meisten der 17 Beiträge. Schließlich ist die Folgerung naheliegend, der (totgesagte) Staat erweise sich als besonders „lebendig" 8 . Andreas Anter bringt den Sachverhalt überzeugend auf den Punkt: Der These vom Verschwinden des Staates liege eine überkommene Staatsidee zugrunde; der Staatsbegriff habe sich als vitales Konzept behauptet. „Staatssicherheit ist auch unter gegenwärtigen Bedingungen eine entscheidende Bedingung für eine freiheitliche Gesellschaft" (S. 27). Und Gunnar Folke Schuppert, Autor eines einschlägigen Werkes ${ }^{9}$, spricht vom sich selbst entmachtenden Leviathan - die meisten

6 Vgl. Jean François Revel, So enden die Demokratien, München / Zürich 1984.

7 Vgl. Francis Fukuyama, Das Ende der Geschichte. Wo stehen wir? München 1992.

8 Vgl. unter anderem Eckhard Jesse (Hrsg.), Renaissance des Staates? (Veröffentlichungen der Deutschen Gesellschaft für Politikwissenschaft, Bd. 29), Baden-Baden 2011.

9 Vgl. Gunnar Folke Schuppert, Staat als Protest. Eine staatstheoretische Skizze in sieben Aufzügen, Frankfurt am Main / New York 2010. 
„Selbstentmachtungen gehen auf das Konto der Exekutive und häufig zu Lasten des Parlaments" (S. 48).

Der erfreulicherweise interdisziplinär gestaltete Sammelband ist in fünf Kapitel gegliedert: Der Staat der modernen Gesellschaft - Europäisierung der Staatlichkeit - Transformation von Wohlfahrtsstaatlichkeit - Politik und Öffentlichkeit im digitalen Zeitalter - Verfassungsstrukturen und Märkte. Der letzte Teil, eher randständig und in sich heterogen, zielt weniger auf die Frage nach der Souveränität des Staates. Die drei Beiträge zur Thematik „Europäisierung und Wandel der Staatlichkeit“ sind besonders aufschlussreich. Prinzipielle Überlegungen vermischen sich mit aktuellen Bestandsaufnahmen. Roland Sturm untersucht den Souveränitätstransfer von den Nationalstaaten zur EU, Enrico Peuker verweist auf die Europäisierung staatlicher Verwaltungen, Maurizio Bach analysiert kritisch die Rolle des Nationalstaates in der EU.

Für diesen Band gilt das Gesagte über Kielmanseggs Essaysammlung. Es mangelt etwas an Geschlossenheit. Sieht Kielmansegg im Nationalstaat eher einen Garanten der Demokratie, so nahmen manche Autoren in dem Sammelwerk gerade in Nationalstaaten postdemokratische Tendenzen wahr.

Eckhard Jesse

\section{Das Bundesverfassungsgerichtsgesetz: kompakte und selbstbewusste Erläuterungen}

\section{Lenz, Christofer und Ronald Hansel: Bundesverfassungsgerichtsgesetz. Handkommentar, Nomos} Verlagsgesellschaft, Baden-Baden 2013, 677 Seiten, € 98,-.

Der Markt für Kommentarliteratur zum Bundesverfassungsgerichtsgesetz ist relativ überschaubar: So gibt es das doppelbändige Loseblattwerk mit knapp 4.000 Seiten, das Theodor Maunz begründet hat und heute von ehemaligen Mitgliedern des Bundesverfassungsgerichts und Hochschullehrern betreut wird, sowie - bislang - die beiden Handkommentare von Hans Lechner und Rüdiger Zuck (6. Auflage 2010, 815 Seiten, $88 €$ ) sowie vom HerausgeberTeam Dieter C. Umbach, Thomas Clemens und Franz-Wilhelm Dollinger (2. Auflage 2005, 1.436 Seiten, $178 €$ ). Dieses Duo der Handkommentare bekommt nun Verstärkung und Konkurrenz durch das neu in erster Auflage erschienene Werk von Christofer Lenz und Ronald Hansel.

Angesichts der großen Bedeutung, die die Verfahren vor dem Bundesverfassungsgericht oftmals haben, ist es nur zu begrüßen, wenn sich das Angebot der dafür einschlägigen prozessrechtlichen Kommentarliteratur verbreitert. Gleichwohl stellt sich bei jedem Werk, das ein literarisch bereits erschlossenes Gebiet betritt, die Frage nach den Alleinstellungsmerkmalen und seinem wissenschaftlichen oder rechtspraktischen Mehrwert. In diesem Kontext ist zunächst einmal das Autorenduo selbst interessant: Während die beiden schon vorhandenen Kurzkommentare jeweils eine „geschlossene Perspektive“ aufweisen, nämlich die Bestimmungen des Verfassungsprozessrechts entweder aus der anwaltlichen Sicht (Lechner) Zuck) oder aus der Perspektive von wissenschaftlichen Mitarbeitern des Gerichts (Umbach) Clemens/Dollinger) erläutern, verbinden Lenz und Hansel diese beiden Perspektiven in einem Werk. Die anwaltliche „Außensicht“ auf das Verfassungsprozessrecht bringt Christofer Lenz ein, der seit 15 Jahren in einer bundesweit agierenden Anwaltskanzlei unter anderem 